\title{
Body area networks and technologies
}

\author{
Laurent Ouvry $\cdot$ Bin Zhen $\cdot$ Simon Cotton
}

Published online: 5 February 2011

(C) Institut Télécom and Springer-Verlag 2011

Body Area Networks (BAN) are formed by networks of devices, which are placed on or in close vicinity of the human body, and in the case of some specialized medical applications, inside the body as implants. Technologies for BANs have gained significant international interest in recent years from researchers both in academia and industry. This has been driven in no small part by the huge potential for commercial exploitation and the prospect of technologically revolutionizing everyday life. The future applications that will benefit from advances in BAN technology include healthcare, fitness, sports, security, and interactive gaming. One key area where BANs are already having a considerable impact is Ambient Assisted Living (AAL). By adopting BAN technologies, AAL has begun to improve quality of life and provide much greater independence for today's aging society. Here, wearable sensors and actuators with a wireless interface are noninvasively worn by the user and wirelessly connected to a gateway, either

\section{Ouvry $(\bowtie)$}

CEA-Leti Minatec Campus,

17 rue des Martyrs,

38054 Grenoble Cedex, France

e-mail: laurent.ouvry@cea.fr

\section{B. Zhen}

Wireless Research Department, Huawei,

Kuike Building, No. 9, Xinxi Rd., Shand-Di, Hai-Dian District,

Beijing 100085, China

e-mail: zhen.bin@huawei.com

\section{S. Cotton}

Royal Academy of Engineering Research Fellow, ECIT Institute,

The Queen's University of Belfast,

Northern Ireland Science Park,

BT3 9DT, UK

e-mail: simon.cotton@qub.ac.uk cellular or internet, to provide real-time health information remotely to medical staff or to other family members.

While much innovation has occurred, a number of critical challenges still exist that will determine the future acceptance and success of BANs. Among these are challenges related to wireless node design such as ease-ofuse, efficiency and reliability, and to the wireless interface such as optimum network topology, protocol, and security. These challenges are made even more difficult to overcome due to the constraints placed on wearable technology when compared to other wireless systems. In particular, the desirable compact, low-form factor of the wireless devices used in BAN applications puts extremely severe limitations on battery size, and thus, power consumption. In addition, badly chosen mounting arrangements and reduction of antenna size can cause decreased efficiency, and thus, lower the quality of the radio link. There are of course other important factors, such as sociological aspects of acceptance; however, they are not discussed here as they fall outside the context of this special issue, which is focused on the technological aspects of BANs. To advance our understanding of these challenges and how they may be surmounted, the content of this special issue addresses the following important questions: How does the BAN propagation channel behave? How should an antenna be designed to be worn on the body? What is a reasonable transmit power to use? What are the feasible receiver performances? What are the best modulation schemes to use to provide good performance, robustness, and sufficient bit rate for different applications? How can the protocol layer guarantee sufficient quality of service and reliability?

Within the last few years, an extensive amount of research has been performed to provide answers to such essential questions, which are regularly posed to the BAN designer. In particular, the IEEE 802.15.6 task group was 
formed in 2007 to create a wireless standard dedicated to BANs. This special issue provides an insight of the cutting edge research currently being conducted worldwide on BAN topics, such as antenna design, the propagation channel, both narrow-band and ultra-wideband (UWB), physical layer technologies, medium access control (MAC) layer, and its evaluation. Furthermore, it presents work from some of those who were involved in the development of the IEEE 802.15.6 standard and other leading researchers in the field.

The issue begins with a paper by Christophe Roblin et al. It studies the aspects related to antenna design in the BAN context. Such important phenomenon like antenna detuning when placed close to the body are demonstrated and design techniques to mitigate such dispersion effects are proposed for the $2.4 \mathrm{GHz}$ band. The role of the antenna as a part of the propagation channel is also shown with a wide comparison in the UWB context.

The second paper is the sequel to the first, and is devoted to the characterizing and modeling the BAN propagation channel. A scenario-based approach, i.e., avoiding the classical distance dependant approach, is used to determine the BAN statistical behavior and models from several measurement campaigns presented. Measurement setups are carefully described in the UWB context and issues related to the measurement uncertainties are described. The numerous sources of variability of the channel are addressed and a particular focus is put on the time variant channel in dynamic situations and its modeling.

Following from this, Attaphongse Taparugssanagorn et al. attempt to expand the knowledge of the UWB channel in the frequency range of $3.1-10 \mathrm{GHz}$ in the presence of a human body. In this paper, the dynamic behavior of the channel is studied through its second-order statistics, namely, the good and bad channel durations as well as the level crossing rate. A suitable model for the design of protocols is proposed, characterized by its low complexity. In addition, the dynamic delay spread of the channel is investigated.

The fourth paper by David Burton Smith et al. is dedicated to the narrowband channel in the $402 \mathrm{MHz}$ Medical Implants Communications Service band and the $900 \mathrm{MHz}$ and $2.4 \mathrm{GHz}$ Industrial, Scientific and Medical (ISM) bands. Dynamic situations are considered, comprising of scenarios which replicate an on-body device communicating with an off-body device. An interesting novel aspect of this paper is the consideration of long sampling durations, i.e., hours of everyday activity. Careful consideration is given to various adult human test subjects who are used to take into account variability. Fade duration and fade depth with direct matching to second-order temporal statistics are also characterized, since they have a considerable importance in the design and evaluation of protocols as already highlighted.

In the next paper by Anda Raluca Guraliuc et al., the focus is on the characterization of the propagation channel between on-body devices operating in the 2.4 and $5.8 \mathrm{GHz}$ ISM bands. Commercial modules and low-profile annular ring slot antennas were used and measurements were performed for different locations on the body. Measurement results have been compared with simulations by using simplified body models of canonical geometries. Characteristic parameters appearing in the propagation models for the path loss have been calculated.

The last two papers concern MAC layer design and evaluations, taking into account an advanced knowledge of BAN channels as provided for instance by some of the previous papers.

Firstly, Paul Ferrand et al. evaluate MAC solutions for typical body-monitoring applications, where a set of devices transmit their data in real time to a common sink also located on the body. The authors show that although the network complies with a star topology, due to the specificity of the dynamic BAN radio channel, the radio links suffer from losses opening the need for multi-hop, relaying or cooperative approaches in an opportunistic manner to improve the overall reliability. The approaches are analyzed from a theoretical point of view and using realistic simulations based upon a time-division multiple access MAC protocol close to IEEE 802.15.4. The simulations use a dynamic BAN channel emulator and the packet error rate outage probability as a performance criterion.

In the final paper of the special issue, Yan Zhang et al. propose a priority-guaranteed MAC protocol to adapt to very diverse application requirements. In this protocol, data channels are separated from control channels to support collision-free high data rate communication for multimedia applications. Priority-specific control channels are adopted to provide a priority guarantee to life-critical medical applications. Traffic-specific data channels are deployed to improve resource efficiency and latency performance. Moreover, an asynchronous wakeup trigger mode is proposed as an enhancement to the priority traffic. Simulation results are provided to demonstrate superiority to IEEE 802.15.4.

Finally, the editors of this issue would like to thank the editor-in-chief and Editorial Board of Annals of Telecom for providing them with opportunity to compile this edition. In addition, the editors are grateful to the authors and effort of the reviewers during the pier review process of all submitted papers. 